\title{
Automatic spatial association for luminance
}

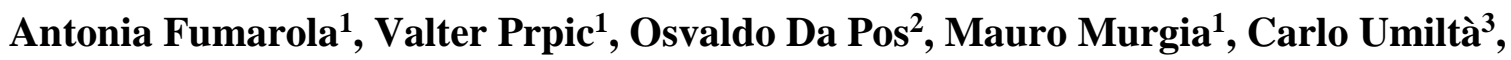 \\ Tiziano Agostini ${ }^{1}$ \\ 1 Department of Life Sciences, University of Trieste, Italy \\ 2 Department of Applied Psychology, University of Padova, Italy \\ 3 Department of General Psychology, University of Padova, Italy
}

Published in Attention, Perception, and Psychophysics

First online: 09 January 2014

\section{Correspondence to:}

Antonia Fumarola

Department of Life Sciences

Via Weiss, 21

34100 Trieste, Italy

PHONE: (+39) 0405582740

E-MAIL: antonia.fumarola@gmail.com 
Space-luminance association

\begin{abstract}
In the present study we investigated whether luminance and side of response execution were associated, showing a SNARC-like effect (faster responses with the left hand for dark stimuli and vice versa). Thirty participants were tested in two experiments. In Experiment 1, the association between space and luminance of chromatic stimuli was directly tested (brightness discrimination). In Experiment 2, the same spatial association was tested indirectly (hue discrimination). Results showed that participants responded faster with their left hand to hues with lower luminance and with their right hand to hues with higher luminance, in either the direct or the indirect task. The consistency of this association in both tasks demonstrates the automaticity of the SNARC-like effect for luminance.
\end{abstract}

Key words: SNARC, luminance, hue, spatial association 


\section{INTRODUCTION}

The Spatial Numerical Association of Response Codes (SNARC) effect is a well-known phenomenon showing a spatial relation between number magnitude and side of response execution (Dehaene, Bossini \& Giraux, 1993). The original study, showed that, in a parity judgment task, participants were faster at processing the parity of large one-digit numbers (e.g., "9" presented in the center of the screen), when responses were executed in the right hemispace, whereas they were faster at judging the parity of smaller numbers (e.g., "1" presented in the center of the screen), when responses were executed in the left hemispace. The SNARC effect suggests that the representations of relatively small magnitudes are spatially compatible with the left hemispace and those of relatively large magnitudes are spatially compatible with the right hemispace. Traditionally, this effect has been explained in terms of a left-to-right oriented mental number line (MNL), even though other authors proposed alternative explanations (see Proctor \& Cho, 2006; Gevers et al., 2006).

This effect has been shown not only for number magnitude, but also for non-numerical ordered sequences. Gevers, Reynvoet, \& Fias (2003) investigated the spatial organization of two non-numerical ordered sequences: months of the year and letters of the alphabet. The authors asked participants to judge whether months presented in the centre of a screen came before or after “June”, and to judge whether letters presented in the centre of a screen came before or after the letter "O”. Results showed that the mental representation of these ordinal sequences could be spatially coded, because the first months of the year were processed faster with responses executed in the left hemispace, whereas the reverse pattern was obtained for the last months of the year. Similar findings were reported in a task employing letters of the alphabet instead months.

SNARC-like effects have been found not only in the visual domain but also in the auditory domain. For instance, Rusconi et al. (2005) explored the spatial representation of pitch height, using both horizontal and vertical response positions, in both direct and indirect tasks. In the direct task, 
non-musicians were asked to judge if a probe tone was higher or lower than a fixed reference tone, by pressing one of two keys with their left of right hand. In the indirect task, participants (nonmusicians and musicians) were exposed to relatively high or low tones, but were asked to classify sounds as being produced by wind or percussion instruments, instead of judging the pitch. Results evidenced a SNARC-like effect, showing that the internal representation of pitch height is spatially organized, especially in participants with formal musical education. This effect has been named Spatial Musical Association of Response Codes (SMARC effect).

Summarizing, the SNARC effect can be found in different domains, suggesting a common mechanisms for representing quantities in the spatial dominion. One of the most relevant attempt to group empirical evidence on magnitude processing was proposed by Walsh (2003) in his “A Theory Of Magnitude” (ATOM). Walsh hypothesized the existence of a common code system processing the magnitude through three dimensions: time, space, and numerosity. Considering the more general concept of magnitude, relatively independent from the specific domain, the ATOM model can explain the SNARC effect also for non-numerical ordered sequences. For this reason, instead of SNARC, Walsh suggested the name SQARC (Spatial Quantity Association of Response Codes).

The ATOM model has many implications regarding the magnitude processing of both spatial and non-spatial dimensions. For instance, according to ATOM, the processing of numerical information and both spatial dimensions (e.g. size) and non-spatial dimensions (e.g. luminance) should cause a mutual interference. In their review, Cohen Kadosh, Lammertyn \& Izard (2008; also see Bonato, Zorzi \& Umiltà, 2012) described similar effect patterns (such as distance, size and SNARC effects) with different kinds of quantities. In particular, they reviewed converging evidences in different domains, such as digits magnitude, geometrical shapes, lines length, pitch height and luminance, consistently with the ATOM model.

Among the above-mentioned dimensions, only a few studies investigated the magnitude representation for luminance and its interactions with other magnitudes. In these studies, luminance 
was mainly investigated in association with digits, by using a congruity paradigm (Algom, Dekel, \& Pansky, 1996; Henik \& Tzelgov, 1982). This paradigm assumes that an irrelevant variable (e.g., number magnitude) can affect the performance on a cognitive task (e.g., physical size judgements). Therefore, if pairs of digits are compared with respect to physical size, the irrelevant variable (e.g., number magnitude) would facilitate the performance in congruent pairs (e.g., 5-8) and impair it in incongruent pairs (e.g., 5-8).

The first study that examined the association between luminance and other magnitudes was performed by Pinel and colleagues (2004). The authors, by using a congruity task, manipulated number, physical size, and luminance within a single stimulus. They asked participants to judge each of the above-mentioned dimensions in separate blocks and found a significant interference between size and luminance, but little or no interference was observed between number and luminance. However, the simultaneous manipulation of number, physical size, and luminance might have masked the association between number and luminance, as suggested by Cohen Kadosh, Cohen Kadosh, \& Henik (2008). Indeed, it has been reported that when manipulating only number and luminance there is an association between these dimensions both at behavioural level and at neuronal level (Cohen Kadosh, Cohen Kadosh, \& Henik, 2008; Cohen Kadosh \& Henik, 2006).

As concerns these two latter works (Cohen Kadosh and colleagues, 2006; 2008), Gebuis \& van der Smagt (2011) noted contrasting results in the direction of the association. Indeed, Cohen Kadosh \& Henik (2006) found faster responses for stimuli that were numerically larger and darker; while Cohen Kadosh, Cohen Kadosh, \& Henik (2008) found faster responses for numerically larger and brighter stimuli. Gebuis \& van der Smagt (2011) hypothesized that this effect could be due to either luminance or luminance contrast, because in previous studies faster responses for numerically larger and darker stimuli were found with a bright background (compared to all the stimuli), and faster responses for numerically larger and brighter stimuli were found with a dark background (compared to all the stimuli). In their study they used a congruity task, comparing either numbers or 
luminance. In the first case, they found that the luminance contrast hypothesis fitted better the results, while in the second case, they found confirmation for the luminance hypothesis.

In general, it seems that there is an association between luminance, numbers and physical size, however the association between luminance and side of response execution is a phenomenon that has been rarely examined. As of now, only two studies investigated the existence of a SNARClike effect for luminance. However, these studies found apparently contrasting results, rendering this effect quite elusive. For this reason, we decided to further investigate this phenomenon.

The first study that addressed this issue, even though it was not its primary aim, was the above-mentioned one of Cohen Kadosh et al. (2008). They failed to report an association between luminance and side of response execution, whereas they confirmed an association between number magnitude and side of response execution (the classical SNARC effect). However, Cohen Kadosh et al. manipulated number magnitude and luminance within a single stimulus and this could have masked a SNARC-like effect for luminance.

The second study, conducted by Ren, Nicholls, Ma \& Chen (2011), investigated SNARClike effects for different types of magnitudes (numerical, physical, luminance, conceptual and sound intensity magnitudes). This is the only study that reported a SNARC-like effect for luminance. Indeed, while the previously described studies manipulated luminance associated with other variables (magnitude and/or physical size) in congruity tasks, Ren et al. asked participants to perform a direct luminance comparison in their third experiment. Participants were exposed to a reference grey disk, followed by a second disk, brighter or darker than the reference one, and were asked to judge if the second disk was darker or brighter than the reference, by pressing a left or a right key. It is important to highlight that both disks were always darker than the grey background. Their results evidenced that responses with the right hand were faster to darker stimuli and responses with the left hand were faster to brighter stimuli.

Based on our review of the available literature, we came to the conclusion that two points 
need to be taken into consideration. The most important one is that Ren et al. reported a SNARClike effect adopting a direct task (luminance comparison), which does not allow us ascertain whether the luminance spatial association is due to an automatic process, as in the classic SNARC effect obtained with an indirect task (parity judgement; Dehaene, Bossini \& Giraux, 1993). The second concerns the hue of the stimuli. All the previous experiment used achromatic stimuli, however it is not known whether the effect found by Ren and colleagues can be replicated with chromatic stimuli. Indeed, Fias, Lauwereyns and Lammertyn (2001) failed to find the SNARC effect in an indirect task based on hue judgment. However, while the task relevant information (hue) was a pre-attentive feature (Treisman \& Gelade, 1980), the irrelevant information (number) was not. Such difference between relevant and irrelevant information may have affected the results.

The first of the above mentioned-considerations is of crucial importance for the present study. Indeed, our purpose was to verify the existence of a relation between luminance and side of response execution, both in direct and indirect SNARC-like tasks. Differently from all the abovementioned studies, we used chromatic instead of achromatic stimuli. This methodological innovation allowed us to introduce a task irrelevant dimension (hue discrimination) in the indirect task. Because we adopted chromatic stimuli, in a first experiment we investigated the effect reported by Ren et al. (2011) by using an analogous direct comparison task for luminance. In a second experiment, we made use of the same chromatic stimuli to explore the automaticity of the association with an indirect task. Differently from Fias, Lauwereyns and Lammertyn (2001), in our case relevant and irrelevant information (hue and brightness) were both pre-attentive features. 


\section{EXPERIMENTS}

\section{Experiment 1: Direct task}

\section{METHOD}

\section{Participants}

Thirty students participated in Experiment 1: fifteen males ( $M=25,7$ years; $S D=2.34)$ and fifteen females ( $M=25,4$ years; $S D=2.87)$. All participants were right-handed and had normal or corrected-to-normal vision.

\section{Apparatus and stimuli}

We used the E-Prime software, version 1.2, to create and control Experiment 1 (http://www.pstnet.com/eprime.cfm). Stimuli were colors, mostly different in hue but similar in luminance (seven unique reds and seven unique greens; see Figure 1). Because our task was to investigate whether reaction times (RTs) to the hue of a color would depend on its luminance, the luminance of the seven colors of a series was different, but it was the same for each pair in the two series. Equiluminance was in a first step colorimetrically determined, but in a second step it was corrected following the minimum distinct border method, as proposed by Boynton (1973). Based on this procedure, the two colors of each pair, in alternating stripes side by side, were visually compared and the luminance of one of them was changed until the border dividing the two colors appeared to be minimally distinct to the observer. The final versions of the colors were specified in the CIELUV $2^{\circ}$ space (suitable to describe self luminous colors), for a luminance close to D65 (see Table 1 and Figure 2).

Colors were presented on a calibrated monitor (Quato Intelli Proof 242 excellence). Each trial 
encompassed the following stimuli: A fixation cross measuring $1 \mathrm{~cm}$ by $1 \mathrm{~cm}$ and a square (red or green) measuring $12.50 \mathrm{~cm}$ by $12.50 \mathrm{~cm}$. All stimuli were presented in the centre of the screen against a black background.

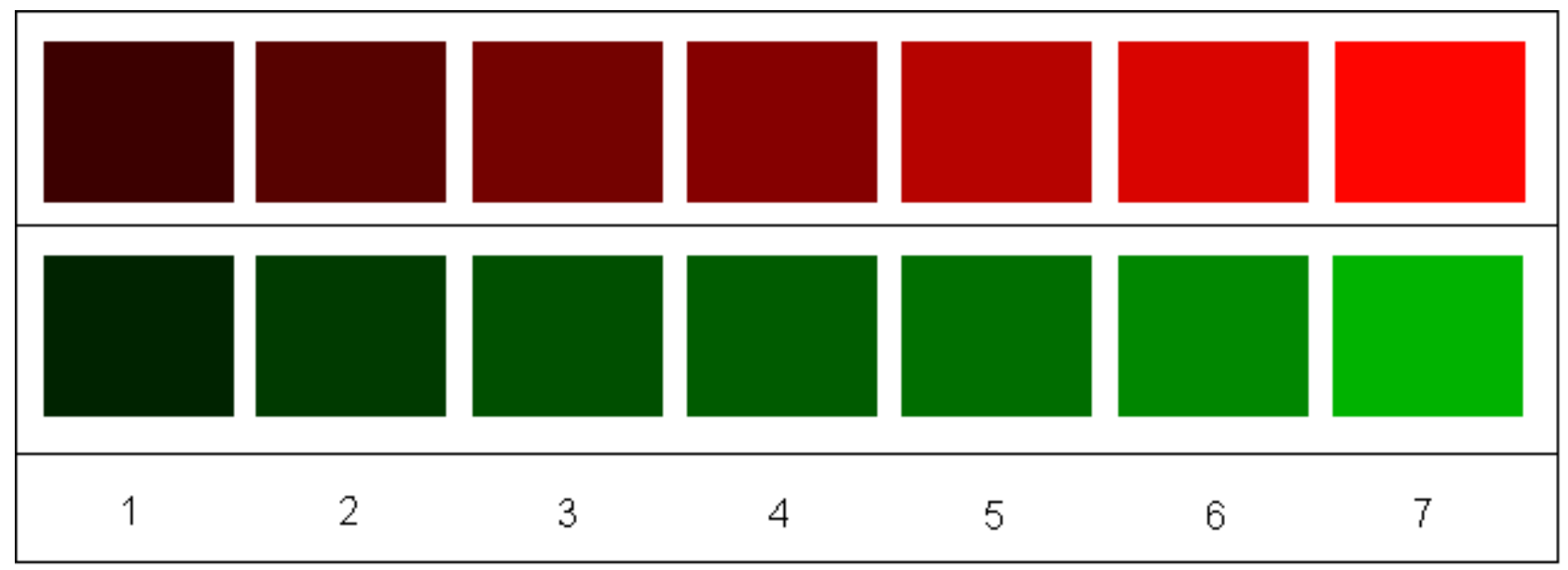

Figure 1. Stimuli

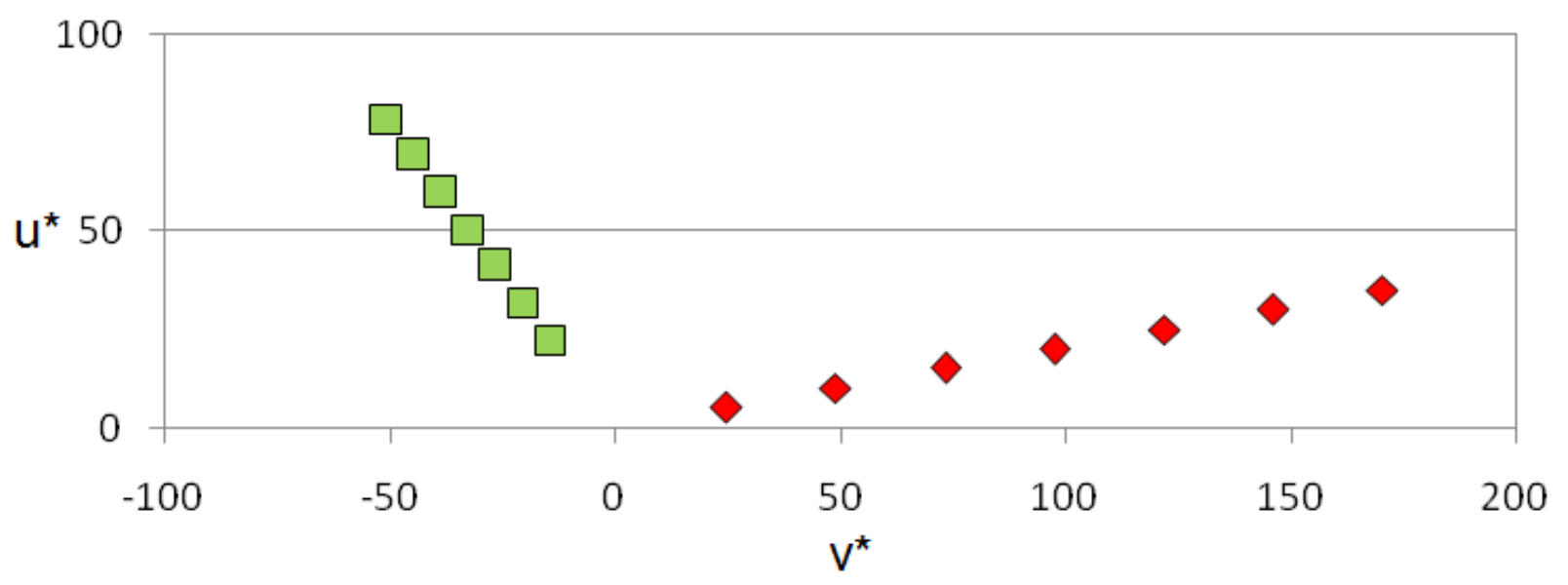

Figure 2. $\mathrm{u}^{*}$ and $\mathrm{v}^{*}$ coordinates of the experimental colors in a CIELUV $2^{\circ}$ diagram. Red diamonds: series of the 7 reds; green squares: series of the 7 greens. 


\begin{tabular}{|c|c|c|c|c|c|}
\hline & & reds & greens & \\
\hline Colour & $\mathrm{L}^{*}$ & $\mathrm{u}^{*}$ & $\mathrm{v}^{*}$ & $\mathrm{u}^{*}$ & $\mathrm{v}^{*}$ \\
\hline 1 & 59 & 170.52 & 35.08 & -50.35 & 78.68 \\
\hline 2 & 50 & 146.25 & 30.09 & -44.36 & 69.32 \\
\hline 3 & 44 & 121.99 & 25.1 & -38.37 & 59.95 \\
\hline 4 & 38 & 97.72 & 20.11 & -32.37 & 50.59 \\
\hline 5 & 32 & 73.46 & 15.11 & -26.38 & 41.22 \\
\hline 6 & 24 & 49.19 & 10.12 & -20.39 & 31.86 \\
\hline 7 & 14 & 24.93 & 5.13 & -14.39 & 22.49 \\
\hline
\end{tabular}

Table1. $\mathrm{L}^{*} \mathrm{u} \mathrm{v}^{*}$ values of the 7 red and 7 green colors used in Experiment 1 and Experiment 2 (Illuminant close to D65). Unit of $\mathrm{L}^{*}=\mathrm{Cd} / \mathrm{m}^{2}$. The value of white was $120 \mathrm{Cd} / \mathrm{m}^{2}$

\section{Procedure}

Experiment 1 took place in a quiet, dimly lit room without environmental distractions. Participants sat in front of the monitor and were asked to put their left index on key " $\mathrm{A}$ ” and the right index on key "L". The viewing distance was $57 \mathrm{~cm}$. Each trial started with a fixation cross displayed at the centre of the screen for 300 ms, followed by a black screen for 130 ms. Afterwards, the reference hue was presented for $1000 \mathrm{~ms}$, followed by an inter-stimulus interval (ISI) of $700 \mathrm{~ms}$. Then, the target square appeared in the centre of the screen for $1000 \mathrm{~ms}$ or until the response was executed. The inter-trial interval (ITI) was 1500 ms (see Figure 3). Participants had to judge whether the displayed hue was more or less bright than the reference hue (luminance level 4; see Figure 1). Half of the hue stimuli were green and the other half was red.

Experiment 1 comprised two sessions. In the first one participants were asked to press the rightmost key with their right index when the hue was brighter than the reference hue, and to press the leftmost key with their left index when the hue was darker than the reference hue. In the second session, the instruction was the opposite (right/darker; left/brighter). There was a short break between sessions and the order of session presentation was counterbalanced across participants. Each session comprised two blocks of trials (training block and experimental block). Each session 
Space-luminance association

started with the training block (12 trials) and after that the experimental block was presented. Each hue was compared five times with the reference hue in the experimental block (60 trials). This resulted in a total of 144 trials. Finally, the order of Experiments 1 and 2 was counterbalanced across participants.

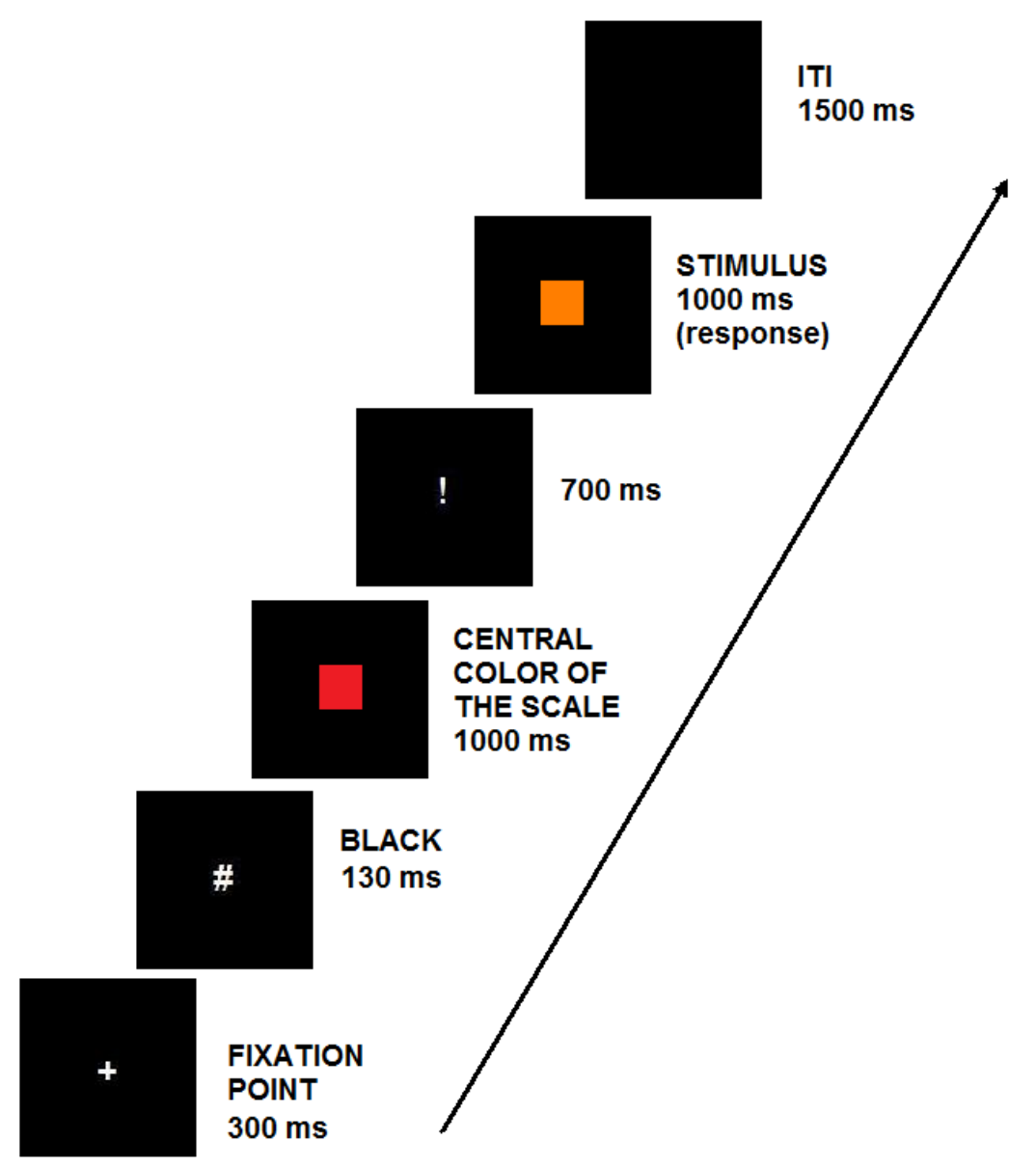

Figure 3. Direct task. Example of trial sequence and timing.

\section{Results}


The data were analysed with a regression analysis for repeated measures (Fias et al., 1996; Lorch \& Myers, 1990). The independent variable was the hue luminance and the dependent variable was the difference between the median RT of the left hand and the median RT of the right hand: $\mathrm{dRT}=\mathrm{RT}$ (right hand) - RT(left hand). A total of $13 \%$ of trials were excluded from the analysis due to the incorrect responses.

In the first step, for each participant the median RT of the correct responses was computed across all levels of luminance, separately for left and right hand responses. Then, dRT was computed by subtracting the median RT of left hand responses from the median RT of right hand responses. In the second step, a regression equation was computed across all levels of luminance for each participant. In the third step, one-sample t-tests were performed to test whether regression beta weights of the group deviated significantly from zero.

The analysis revealed that the regression slopes (regression beta coefficients) were significantly different from zero, $t(29)=8.220, p<.001$. More precisely, participants were faster in processing lower luminance stimuli with their left effector, and in processing higher luminance stimuli with their right effector (see Figure 4). 


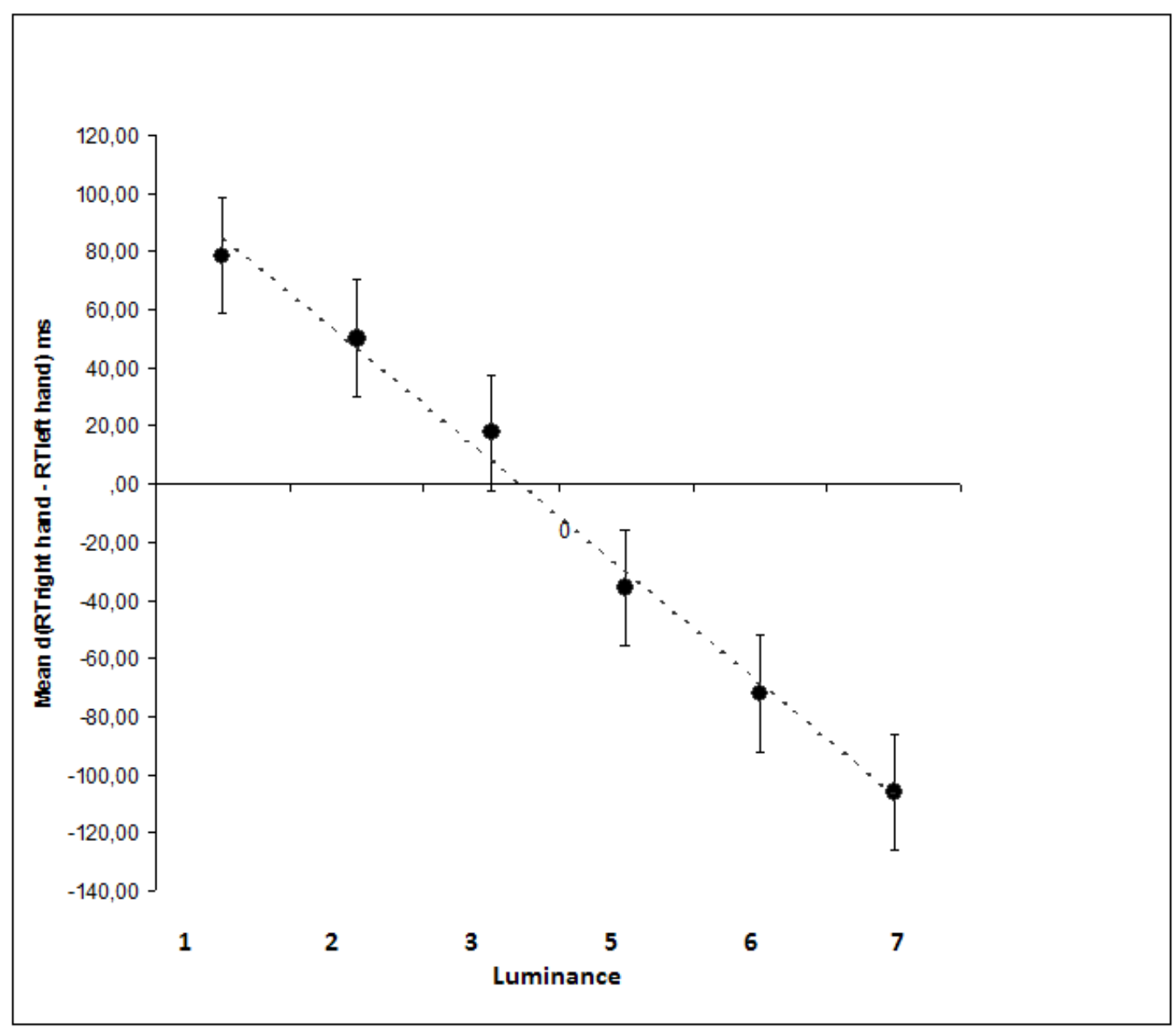

Figure 4. Mean differences $( \pm 2 S E M)$ of the median RT right hand - median RT left hand for the direct task. Positive values indicate faster left hand responses; negative values indicate faster right hand responses.

\section{Discussion}

Our results confirm the spatial luminance association obtained by Ren et al. (2011), also for chromatic stimuli. However, we found that the spatial luminance association is reversed compared with the findings of Ren et al., probably because we used a background darker than the stimuli, rather than brighter. Indeed, our results show that luminance is spatially represented as dark-left and bright-right (as hypothesized also by Cohen Kadosh and colleagues, 2008). Moreover, these results were obtained with a direct task, where participants were explicitly required to judge luminance. However, it is not clear whether the spatial quantity association for luminance is automatic or 
induced by the direct task. To further investigate this phenomenon, we ran a second experiment using an indirect method.

\section{Experiment 2: Indirect task}

\section{METHOD}

\section{Participants}

The participants were the same as those of Experiment 1.

\section{Apparatus and stimuli}

The apparatus and the stimuli were the same as those of Experiment 1.

\section{Procedure}

The procedure was similar to that adopted in Experiment 1. Each trial started with a fixation cross displayed for $300 \mathrm{~ms}$, followed by a black screen for $130 \mathrm{~ms}$. Then the target square appeared in the centre of the screen for $1000 \mathrm{~ms}$ or until the response was executed (see Figure 5). Participants were asked to judge, by pressing one of two keys (A or L), whether the hue of the square was red or green. The inter-trial interval (ITI) was $1500 \mathrm{ms.}$

Experiment 2 comprised two sessions. In the first session, participants were asked to press the rightmost key (L) with their right index when the square was red and the leftmost key (A) with their left index when the square was green. In the second session, the instruction was the opposite (right/green; left/red). There was a short break between sessions and the order of sessions was 
counterbalanced across participants. Each session comprised two blocks of trials (training block and experimental block). Each session started with the training block of 12 trials, followed by the experimental block. In the experimental block each of the 12 hues (6 green and 6 red) was presented five times in random order (60 trials). This resulted in a total of 144 trials.

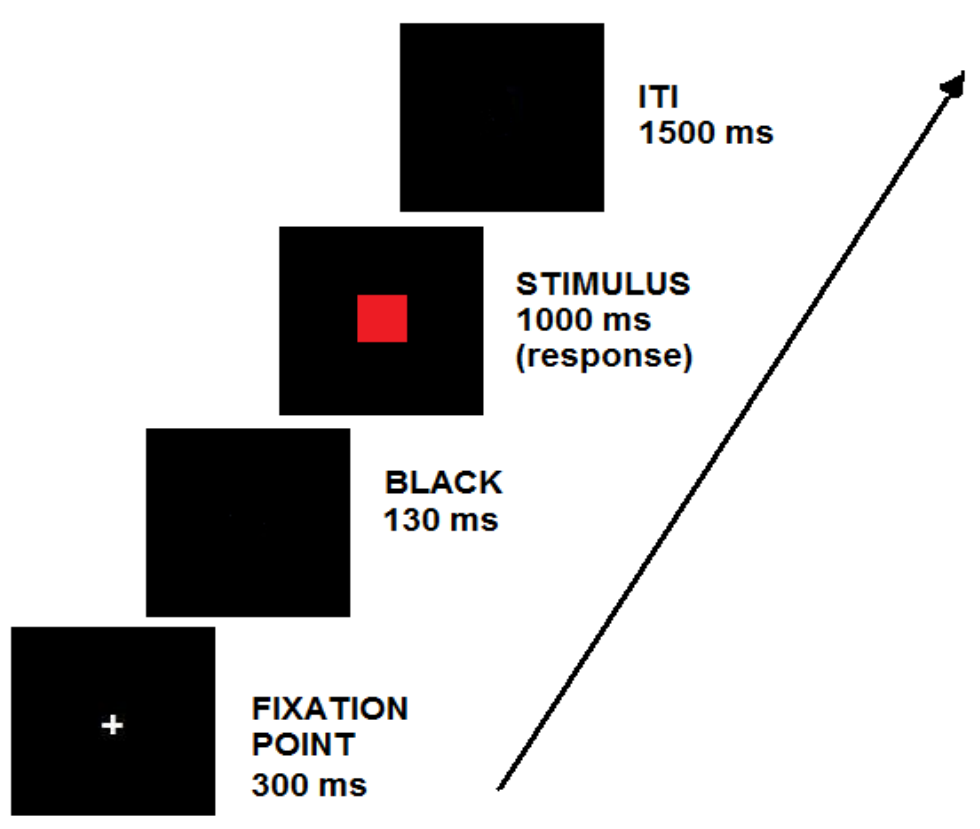

Figure 5. Indirect task. Example of trial sequence and timing.

\section{Results}


Space-luminance association

Data analyses were the same as those carried out in Experiment 1 . A total of $6 \%$ of trials were excluded from the analysis due to the incorrect responses. The analyses revealed that the regression slopes (regression beta coefficients) were significantly different from zero, $t(29)=7,771, p<.001$. There was a left effector advantage in processing hues with smaller luminance and a right effector

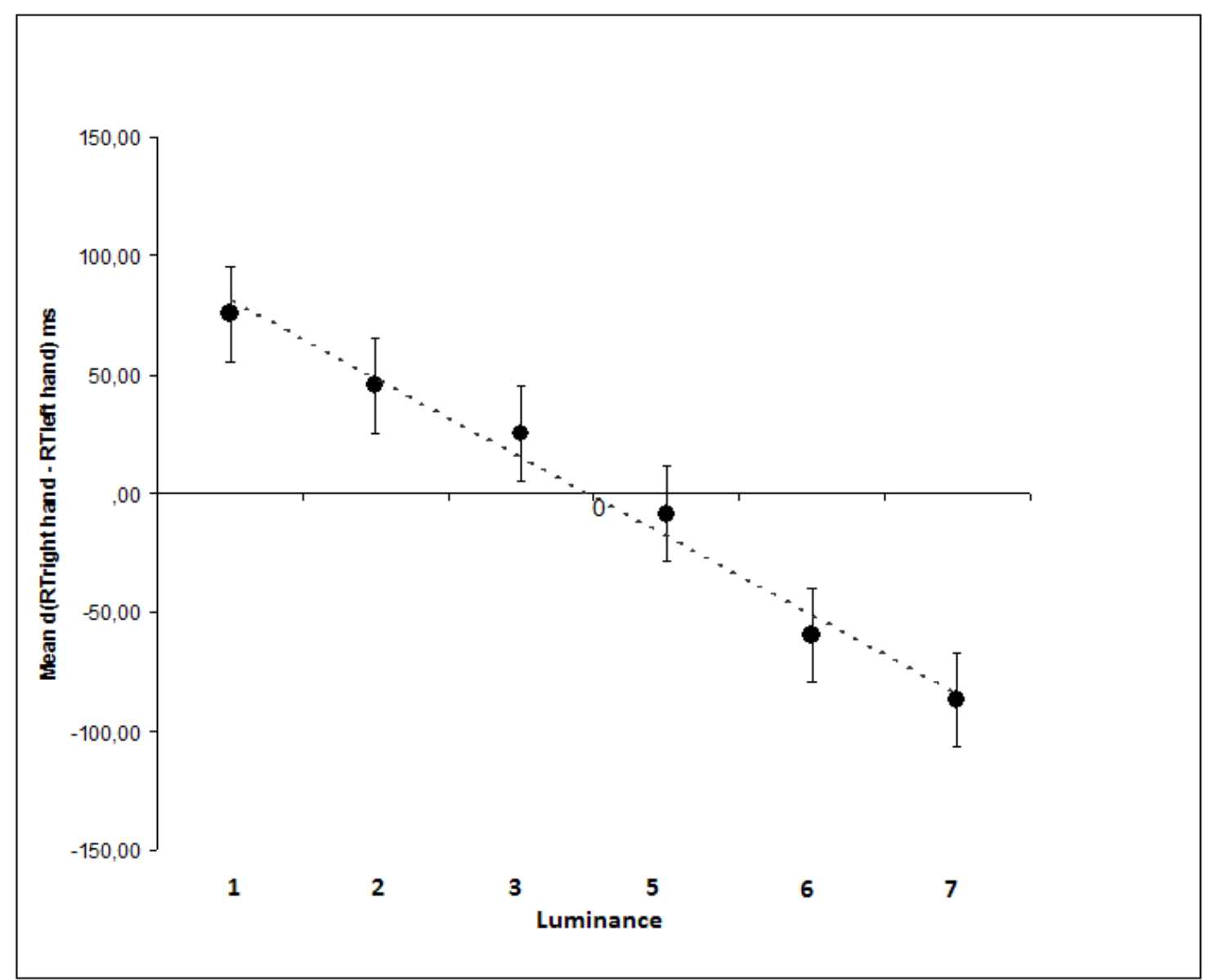
advantage in processing hues with larger luminance (see Figure 6).

Figure 6. Mean differences $( \pm 2 S E M)$ of the median RT right hand - median RT left hand for the indirect task. Positive values indicate faster left hand responses; negative values indicate faster right hand responses. 


\section{Discussion}

The results of the second experiment confirm those obtained in the first one. Indeed, using the same stimuli and background as in the first experiment, the spatial luminance association (dark/left and bright/right) was verified with the indirect task as well. This result proves the automaticity of smaller luminance/left hemispace and larger luminance/right hemispace associations when the background is darker than the stimuli.

\section{GENERAL DISCUSSION}

The aim of the present study was to investigate the existence of a relation between luminance and side of response execution, both in direct and indirect tasks. We tested this hypothesis using a SNARC-like paradigm, where participants had to directly judge the luminance in a comparison task (Experiment 1) and to discriminate the stimuli hue in an indirect task (Experiment 2). Consistently with our expectations, we found a spatial-luminance association. In particular, participants showed a left hand advantage in processing low luminance and a right hand advantage in processing higher luminance, in both direct and indirect tasks.

The most important result of the present study is the evidence of the automatic association between luminance and space. Indeed, even when the task did not require a direct discrimination between luminance quantities, we found a bias in participants' response times between left and right hands. It means that the effect is not due to the mere instructions given to the participants, like it could be questioned in the first experiment of the present work and in the third experiment of Ren et al. (2011), but we proved that this effect is automatic and task-independent. However, the direction of this association seems to be influenced by factors others than the requests of the direct and indirect tasks.

Indeed, the direction of the association described in both our experiments was the opposite of that found by Ren et al. (2011) and is apparently contradictory with their results. However, as Gebuis \& van der Smagt (2011) noted, background luminance can play a role in reversing the 
direction of a number/luminance association. Thus, this is likely to be the most plausible explanation for the different direction of our and Ren et al.'s results. Indeed, whereas Ren and colleagues used a background brighter than the stimuli, in the present study we used a background darker than the stimuli. In any case, we cannot be certain that, by reversing the luminance of the background, the direction of the spatial luminance association would be the opposite. In fact, we did not explore such a possible reversal of the effect.

A second point we addressed in this study concerns the hue of the stimuli. Indeed, whereas previous experiments had used achromatic stimuli, for the first time we introduced chromatic stimuli, allowing us to indirectly study a SNARC-like association for luminance. Differently from Fias, Lauwereyns and Lammertyn (2001), we found a SNARC-like effect using a hue judgement task. We can speculate that these different outcomes may be due to the different processing levels of relevant and irrelevant information (pre-attentive and semantic in the earlier study; both preattentive in the present study). However, further research is needed to better investigate our hypothesis.

As regards the theoretical aspects, our findings demonstrate that the luminance is spatially coded as other types of magnitudes (such as numbers, physical size, pitch, etc.). For this reason, the outcome of the present study provides empirical evidences supporting the ATOM model, and is in line with the idea that spatial representation might be the most suitable form for representing various types of magnitudes (Walsh, 2003). Therefore, our empirical evidences confirm the idea of a general mechanism, independent of the magnitude type, and are consistent with the SQUARC (Spatial Quantity Association of Response Codes) effect proposed by Walsh.

In conclusion, we further confirmed the spatial association of response codes for luminance, extending the knowledge in the dominion of the SNARC-like effects. In particular, the innovations of the present study regard the demonstration that this association is automatic and taskindependent, and is not limited to the achromatic colors. Indeed, for the first time it has been 
consistently demonstrated a spatial luminance association both in a direct and in an indirect tasks, confirming the strength of this effect.

\section{REFERENCES}

Algom, D., Dekel, A., \& Pansky, A. (1996). The perception of number from the separability of the stimulus: The Stroop effect revisited. Memory \& Cognition, 24, 557-572.

Bonato, M., Zorzi, M., \& Umiltà, C. (2012). When time is space: Evidence for a mental time line. Neuroscience and Biobehavioral Reviews, 36, 2257-2273.

Boynton, R.M. (1973). Implications of the minimally distinct border. Journal of the Optical Society of America, 63, 1037-1043.

Cohen Kadosh, R., Cohen Kadosh, K., \& Henik, A. (2008). When brightness counts: The neuronal correlate of numerical-luminance interference. Cerebral Cortex, 18, 337-343.

Cohen Kadosh, R., \& Henik, A. (2006). A common representation for semantic and physical properties: A cognitive-anatomical approach. Experimental Psychology, 53, 87-94.

Dehaene, S., Bossini, S., \& Giraux, P. (1993). The mental representation of parity and number magnitude. Journal of Experimental Psychology: General, 122, 371-396.

Fias, W., Brysbaert, M., Geypens, F., \& d’Ydewalle, G. (1996). The importance of magnitude information in numerical processing: Evidence from the SNARC effect. Mathematical Cognition, 2(1), 95-110.

Fias, W., Lauwereyns , J., \& Lammertyn , J. (2001). Irrelevant digits affect feature-based attention depending on the overlap of neural circuits. Cognitive Brain Research, 12 (3), 415423. 
Gebuis, T., \& van der Smagt, M.J. (2011). Incongruence in number-luminance congruency effects. Attention, Perception, \& Psychophysics, 37, 259-265.

Gevers, W., Reynvoet, B., \& Fias, W. (2003). The mental representation of ordinal sequences is spatially organized. Cognition, 87, B87-B95.

Gevers, W., Verguts, T., Reynvoet, B., Caessens, B., \& Fias, W. (2006). Numbers and space:Acomputational model of the SNARC effect. Journal of Experimental Psychology: Human Perception and Performance, 32, 32-44.

Henik, A., \& Tzelgov, J. (1982). Is three greater than five: The relation between physical and semantic size in comparison tasks. Memory \& Cognition, 10, 389-395.

Lorch, R.F., \& Myers, J.L. (1990). Regression-analyses of repeated measures data in cognitive research. Journal of Experimental Psychology: Learning, Memory, and Cognition, 16, 149157.

Pinel, P., Piazza, M., Le Bihan, D., \& Dehaene, S. (2004). Distributed and overlapping cerebral representations of number, size, and luminance during comparative judgments. Neuron, 41, 983-993.

Proctor, R.W., \& Cho, Y.S. (2006). Polarity correspondence: A general principle for performance of speeded binary classification tasks. Psychological Bulletin, 132, 416-442.

Ren, P., Nicholls, M.E.R., Ma, Y., \& Chen, L. (2011). Size matters: non numerical magnitude affects the spatial coding of response. Plos One, 6(8), e23553

Rusconi, E., Kwan, B., Giordano, B., Umiltà, C., \& Butterworth, B. (2006). Spatial representation of pitch height: the SMARC effect. Cognition, 99, 113-129.

Treisman, A. M., \& Gelade, G. (1980) A feature-integration theory of attention. Cognitive Psychology, 12, 97-136. 
Space-luminance association

Walsh, V. (2003). A theory of magnitude: common cortical metrics of time, space and quantity. Trends in Cognitive Sciences, 7, 483-488. 